Anaesthesist 2015 - 64:463-468

DOI 10.1007/s00101-015-0033-7

Online publiziert: 30. Mai 2015

c) Springer-Verlag Berlin Heidelberg 2015

B. Jüttner ${ }^{1} \cdot$ C. Wölfel ${ }^{2} \cdot$ H. Liedtke ${ }^{3} \cdot$ K. Meyne $^{4} \cdot$ H. Werr ${ }^{5} \cdot$ T. Bräuer $^{6}$. M. Kemmerer ${ }^{7} \cdot$ G. Schmeißer ${ }^{8} \cdot$ T. Piepho $^{9} \cdot$ O. Müller ${ }^{10} \cdot$ H. Schöppenthau ${ }^{11}$

${ }^{1}$ Klinik für Anästhesiologie und Intensivmedizin, Medizinische Hochschule Hannover, Hannover, Deutschland

${ }^{2}$ Anästhesie/Schmerztherapie, SRO Spital Langenthal, Langenthal, Schweiz

${ }^{3}$ Klinik für Anästhesie, Intensivmedizin, Notfallmedizin und Schmerztherapie,

Krankenhaus St. Elisabeth und St. Barbara, Halle, Deutschland

${ }^{4}$ Verband Deutscher Sporttaucher e. V., Goslar, Deutschland

${ }^{5}$ Schifffahrtmedizinisches Institut der Marine, Kronshagen, Deutschland

${ }^{6}$ Berufsgenossenschaft der Bauwirtschaft (BG Bau), Neumünster, Deutschland

${ }^{7}$ Druckkammerzentren RMT GmbH, Wiesbaden, Deutschland

${ }^{8}$ Institut für Arbeit und Gesundheit der DGUV, Deutsche Gesellschaft für

Arbeitsmedizin und Umweltmedizin e. V. (DGAUM), Dresden, Deutschland

${ }^{9}$ Klinik für Anästhesiologie, Universitätsmedizin der Johannes Gutenberg-Universität Mainz, Mainz, Deutschland

${ }^{10}$ Vivantes-Klinikum Friedrichshain, Zentrum für hyperbare Sauerstofftherapie und Tauchmedizin, Berlin, Deutschland

${ }^{11}$ Abteilung für Anästhesie und anästhesiologische Intensivmedizin, Druckkammerzentrum-HBO, Berufsgenossenschaftliche Unfallklinik Murnau, Murnau am Staffelsee, Deutschland

\title{
Diagnostik und Behandlung von Tauchunfällen
}

\section{Neue „Leitlinie Tauchunfall 2014-2017“}

\section{Zusatzmaterial online}

Dieser Beitrag enthält die vollständige "Leitlinie Tauchunfall 2014-2017“" sowie den Leitlinienreport zu der methodischen Vorgehensweise bei der Entwicklung der Leitlinie. Dieses Supplementary Material finden Sie unter DOI 10.1007/s00101-0150033-7.

\section{Einführung}

Die Leitlinie Tauchunfall wurde nach der ersten Veröffentlichung 2002 für den nächsten Gültigkeitszeitraum von 2014 bis Oktober 2017 von einer Expertengruppe überarbeitet und aktualisiert. Die Gesellschaft für Tauch- und Überdruckmedizin (GTÜM) als federführende Fachgesellschaft konstituierte hierzu eine Leitliniengruppe unter Beteiligung der Schweizerischen Gesellschaft für Unterwasser- und Hyperbarmedizin (SUHMS), der Deutschen Interdisziplinären Vereinigung für Intensiv- und Notfallmedizin (DIVI), Sektion Hyperbarmedizin, des Verbands
Deutscher Sporttaucher e. V. (VDST), des Schifffahrtmedizinischen Instituts der Marine, der Berufsgenossenschaft der Bauwirtschaft (BG Bau), des Verbands Deutscher Druckkammerzentren (VDD) und der Deutschen Gesellschaft für Arbeitsmedizin und Umweltmedizin (DGAUM).

Die Leitlinie wurde auf der Basis einer strukturierten Konsensfindung (Entwicklungsstufe S2k) unter der methodischen Begleitung der AWMF - Arbeitsgemeinschaft der Wissenschaftlichen Medizinischen Fachgesellschaften erstellt [1].

Die „Leitlinie Tauchunfall 2014-2017“ soll eine Hilfe für medizinische Entscheidungsprozesse im Rahmen einer leistungsfähigen Versorgung der Patienten darstellen. Basierend auf den Prinzipien der Rettungskette gibt sie Laien, medizinischem Assistenzpersonal und Ärzten in einem spezifischen Notfallbereich Informationen und Instruktionen über den aktuellen Stand der Diagnostik und der Behandlung von Tauchunfällen, den Transport verunfallter Taucher, die erste hyper- barmedizinische Therapie und die weitere medizinische Versorgung. Die Zielsetzung, u. a. dem Laien notfallmedizinische Empfehlungen darzustellen, bedingt die dementsprechend verständlichen Formulierungen.

Die Strukturierung der Leitlinie wurde an den Versorgungsablauf von Tauchunfällen angepasst.

\section{Infobox 1 Taucherärztliche Tele-} fonberatungen

- Nationale DAN-Hotline für Deutschland und Österreich: 00800326668783 (00800 DAN NOTRUF)

- Nationale DAN-Hotline für die Schweiz (via REGA): +41 333333333 (oder 1414 für Anrufe innerhalb der Schweiz)

- VDST-Hotline: +496980088616

- Ansprechstelle des Schifffahrtmedizinischen Instituts der Marine: +49 4315409 1441

- Taucherhotline von aqua med: +49700 34835463

- Internationale DAN-Hotline: +39064211 8685 oder 5685 


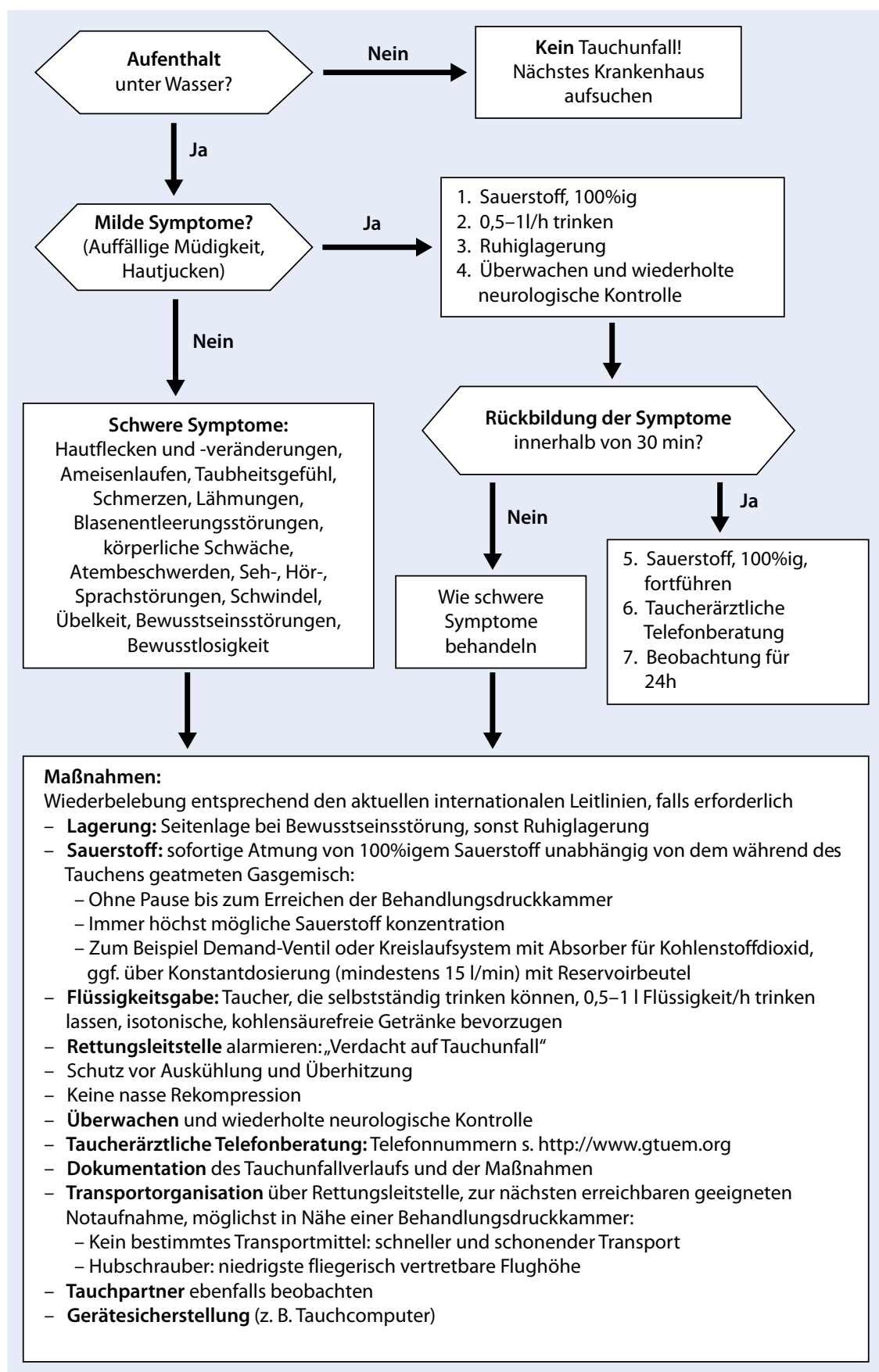

Abb. $1 \Delta$ Primärversorgung bei Tauchunfällen

\section{Diagnostik}

Die bisherige Klassifizierung des Schweregrads von Tauchunfällen in milde Symptome (Fatigue, Hautjucken) und schwere Symptome wurde beibehalten. Dies obwohl international regelhaft schmerzhafte Extremitäten, Hautveränderungen, lokale Schwellungen und geringgradige periphere sensorische Störungen ebenfalls den milden Symptomen zugeordnet wer- der Erstversorgung eine frühzeitige und wiederholte neurologische Untersuchung durchgeführt werden, um eine notwendige Rekompressionsbehandlung nicht ungerechtfertigt auszuschließen. Im Hinblick auf die Anwendung durch Laien wurde der „5-Minuten-Neurocheck“ in den Anlagen der Leitlinie beibehalten. Eine Therapieentscheidung sollte grundsätzlich in Abstimmung mit einem erfahrenen Tauchmediziner getroffen werden (- Infobox 1).

Die Diagnose einer Dekompressionserkrankung kann nur klinisch gestellt werden. Eine bildgebende oder laborchemische Diagnostik ist routinemäßig vor einer HBO nicht erforderlich. Die Überwachung und das Monitoring erfolgen nach den grundsätzlichen Verfahren notfallmedizinischer Standards. Eine weitergehende bildgebende Diagnostik kann zur Differenzialdiagnose von Begleiterkrankungen oder Einschätzung weiterer Verletzungen, wie Pneumothorax, pulmonale Schädigung nach Ertrinkungsunfall oder zentralnervöse Ischämien, sinnvoll sein, wenn dies ohne relevanten Zeitverlust möglich ist [7].

Für die Diagnose eines Pneumothorax wurde die thorakale Sonographie als Untersuchungsmethode in der Leitlinie neu aufgeführt.

Eine weitere radiologische Diagnostik nach der ersten oder weiteren Rekompressionsbehandlungen soll nur bei entsprechender klinischer Symptomatik durchgeführt werden. Der direkte Nachweis von Gasblasen ist ohnehin nur selten möglich und ohne prognostische Bedeutung. Diesbezüglich war die Magnetresonanztomographie bisher der Computertomographie für den Nachweis von Schädigungen des Rückenmarks bei Dekompressionserkrankungen nicht überlegen [8].

Für die Befunddokumentation und die Verlaufsbeurteilung erscheinen neben der fachneurologischen Untersuchung v. a. Verfahren der neurologischen Elektrophysiologie geeignet (z. B. Nervenleitgeschwindigkeit, evozierte Potenziale).

\section{Primärversorgung}

Die präklinische Behandlung orientiert sich grundsätzlich an den symptomorientierten notfallmedizinischen Standards. 
Wesentliche Bedeutung bei der Versorgung von Tauchunfällen hat unverändert die frühzeitige und konsequente Atmung von $100 \%$ igem Sauerstoff $[9,10]$. Nur über einen größtmöglichen Gradienten kann der erhöhte Anteil des Inertgases (z. B. Stickstoff) im Gewebe über die alveoläre Ventilation reduziert werden. Ein gesteigerter Sauerstoffpartialdruck kann zudem die Resorption von intravaskulären Gasblasen verbessern und Gewebehypoxien durch embolisierende Gasblasen sowie deren mechanische und biochemische Folgeschäden vermindern.

In der neuen Leitlinie werden die technischen Möglichkeiten für eine Applikation von $100 \%$ igem Sauerstoff explizit dargestellt. Insbesondere die rettungsdienstlich zunehmende Verfügbarkeit der „Continuous-positive-airway-pressure"(CPAP)-Atmung wurde in die Leitlinie aufgenommen. Die unerwünschte Nebenwirkung, bei verletztem Lungenparenchym durch einen erhöhten Atemwegsdruck einen Pneumothorax zu vergrößern oder zu provozieren, wurde ausführlich und kontrovers in der Leitlinienentwicklung diskutiert. Nach der klinischen Einschätzung der Autoren der Leitlinie wurde der Verdacht für einen Pneumothorax als relative Kontraindikation für die Anwendung einer CPAP-Atmung durch medizinisches Fachpersonal bewertet.

In der Primärversorgung wird unverändert eine moderate Flüssigkeitsgabe mit 0,5-1 1/h empfohlen. Vor dem Hintergrund der Leitlinienanwendung unabhängig der national dichten Rettungsdienststruktur, ist weiterhin die orale Gabe von isotonischen, kohlensäurefreien Getränken bei stabilen, wachen Patienten sinnvoll. Für die Versorgung durch medizinisches Fachpersonal soll der Flüssigkeitsersatz nach notfallmedizinischen Standards mit balancierten i.v.-Vollelektrolytlösungen durchgeführt werden. In diesem Zusammenhang wurde auch das sehr selten auftretende und bisher beschriebene Lungenödem bei Geräte- und auch bei Apnoetauchern diskutiert $[11,12]$. Die Behandlung dieser schwer erkrankten, hoch symptomatischen Patienten entspricht hier der üblichen Notfalltherapie von Lungenödemen anderer Ursachen durch den Rettungsdienst.

Anaesthesist 2015 - 64:463-468 DOI 10.1007/s00101-015-0033-7

(c) Springer-Verlag Berlin Heidelberg 2015

B. Jüttner · C. Wölfel · H. Liedtke · K. Meyne · H. Werr · T. Bräuer · M. Kemmerer · G. Schmeißer · T. Piepho $\cdot$ O. Müller $\cdot H$. Schöppenthau

\section{Diagnostik und Behandlung von Tauchunfällen. Neue „Leitlinie Tauchunfall 2014-2017"}

\section{Zusammenfassung}

Die überarbeitete „Leitlinie Tauchunfall 20142017" wurde im April 2015 von der Gesellschaft für Tauch- und Überdruckmedizin (GTÜM) und der Schweizerischen Gesellschaft für Unterwasser- und Hyperbarmedizin (SUHMS) veröffentlicht. In Zusammenarbeit mit Vertretern der Deutschen Interdisziplinären Vereinigung für Intensiv- und Notfallmedizin (DIVI), Sektion Hyperbarmedizin, dem Verband Deutscher Sporttaucher e. V. (VDST), dem Schifffahrtmedizinischen Institut der Marine, der Berufsgenossenschaft der Bauwirtschaft (BG Bau), dem Verband Deutscher Druckkammerzentren (VDD) sowie der Deutschen Gesellschaft für Arbeitsmedizin und Umweltmedizin (DGAUM) wurde die Leitlinie auf der Basis einer strukturierten Konsensfindung aktualisiert (Entwicklungsstufe S2k). Das Leitlinienprojekt wurde von der Arbeitsgemeinschaft der Wissenschaftlichen Medizinischen Fachgesellschaften (AWMF) methodisch begleitet. Die „Leitlinie Tauchunfall 2014-2017" soll Laien, medizinischem Assistenzpersonal und Ärzten in einem spezifischen Notfallbereich Informa- tionen und Instruktionen über den aktuellen Stand der Diagnostik und der Behandlung von Tauchunfällen geben. Dieser Beitrag fasst Empfehlungen, Zielsetzungen und die wesentlichen Schlüsselempfehlungen der "Leitlinie Tauchunfall" zusammen. Wesentliche Bedeutung bei der Versorgung von Tauchunfällen hat die frühzeitige Atmung von $100 \%$ igem Sauerstoff. Weiterhin werden die Ruhiglagerung und eine moderate Flüssigkeitsgabe empfohlen. Die hyperbare Sauerstofftherapie (HBO) ist bei schweren Dekompressionsunfällen unverändert ohne therapeutische Alternative. Als Behandlungsschema wird grundsätzlich eine hyperbare Oxygenation bei $280 \mathrm{kPa}$ empfohlen. Zukünftig sollte die Etablierung eines bundesweiten Registers von HBO-Notfallbehandlungen zur Qualitätssicherung angestrebt werden.

Schlüsselwörter

Dekompressionserkrankung · Sauerstoff . Hyperbare Sauerstofftherapie .

Patientenlagerung · Flüssigkeitstherapie

\section{Diagnosis and treatment of diving accidents. New German guidelines for diving accidents 2014-2017}

\section{Abstract}

In 2015 the German Society for Diving and Hyperbaric Medicine (GTÜM) and the Swiss Underwater and Hyperbaric Medical Society (SUHMS) published the updated guidelines on diving accidents 2014-2017. These multidisciplinary guidelines were developed within a structured consensus process by members of the German Interdisciplinary Association for Intensive Care and Emergency Medicine (DIVI), the Sports Divers Association (VDST), the Naval Medical Institute (SchiffMedinst), the Social Accident Insurance Institution for the Building Trade (BG BAU), the Association of Hyperbaric Treatment Centers (VDD) and the Society of Occupational and Environmental Medicine (DGAUM). This consensus-based guidelines project (development grade $\$ 2 \mathrm{k}$ ) with a representative group of developers was conducted by the Association of Scientific Medical Societies in Germany. It provides information and instructions according to up to date evidence to all divers and other lay persons for first aid recommendations to physician first responders and emergency physicians as well as paramedics and all physicians at therapeutic hyperbaric chambers for the diagnostics and treatment of diving accidents. To assist in implementing the guideline recommendations, this article summarizes the rationale, purpose and the following key action statements: on-site $100 \%$ oxygen first aid treatment, still patient positioning and fluid administration are recommended. Hyperbaric oxygen (HBO) recompression remains unchanged the established treatment in severe cases with no therapeutic alternatives. The basic treatment scheme recommended for diving accidents is hyperbaric oxygenation at $280 \mathrm{kPa}$. For quality management purposes there is a need in the future for a nationwide register of hyperbaric therapy.

\section{Keywords}

Decompression sickness · Oxygen . Hyperbaric oxygen therapy · Patient positioning $\cdot$ Fluid therapy 


\section{Überlegungen vor Beginn einer Druckkammerbehandlung}

$\begin{array}{lll}\text { - Ausschluss bzw. Drainage eines Pneumothorax? } & \text { - Parazentese? } & \text { - Kontinuierliche "Cuff"-Druck-Kontrolle } \\ \text { - Blasenkatheter? } & \text { - Sicherer venöser Zugang? } & \text { Endotrachealtubus? }\end{array}$

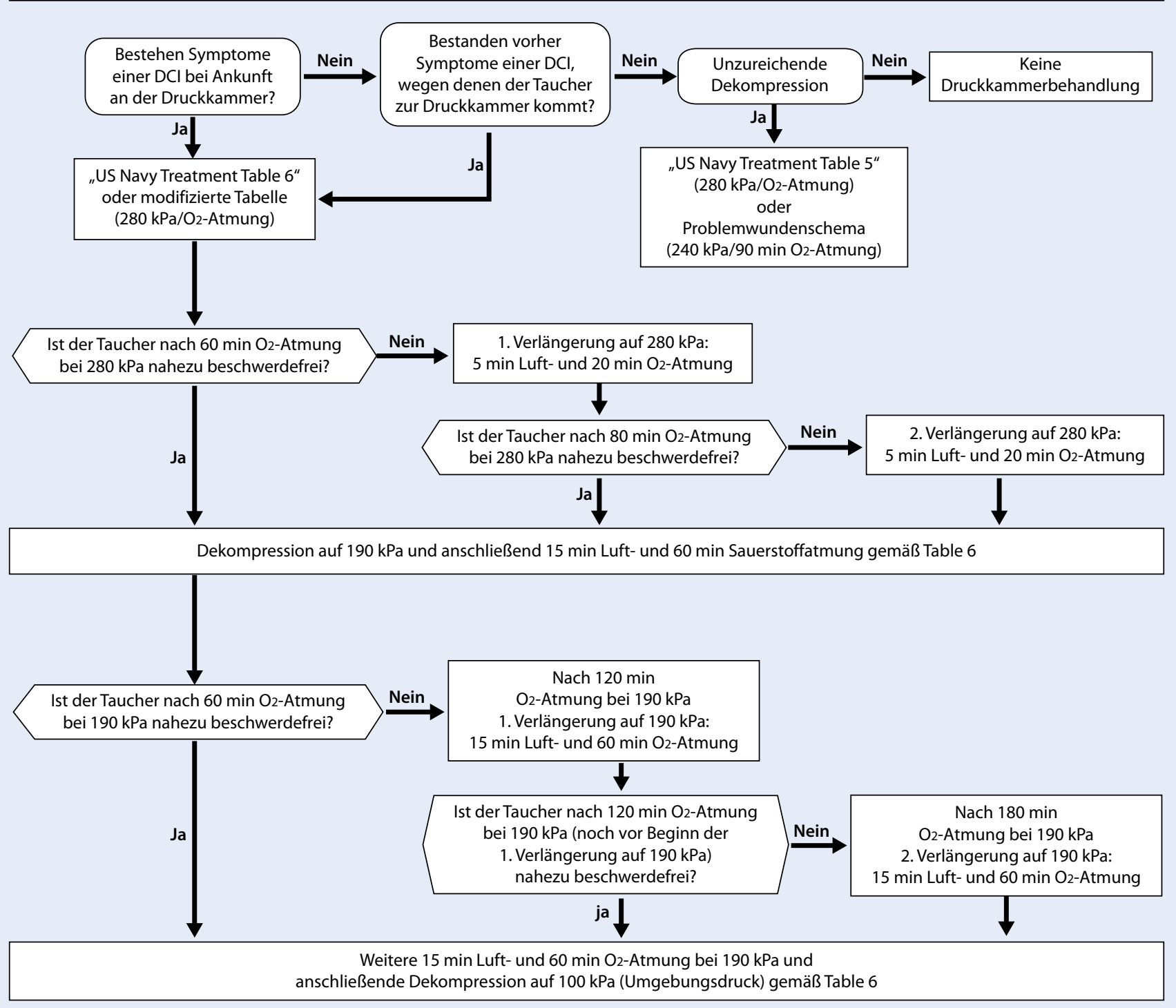

Abb. $2 \Delta$ Erste Druckkammerbehandlung bei Tauchunfällen

Die internationalen Empfehlungen für die Lagerung des Opfers eines schweren Tauchunfalls basieren ursprünglich auf tierexperimentellen Studien [13, 14]. In diesen Untersuchungen wurde ein erhöhtes Risiko für zerebrale Embolien in sitzender Position dargestellt. Andererseits besteht weltweit übereinstimmender Konsensus, keine Kopftieflagerung zu empfehlen [3]. Diese beeinträchtigt den venösen Abfluss und erhöht das Risiko eines Hirnödems.
Aus Sicht der Autoren der Leitlinie ergibt sich unabhängig von der aktuellen wissenschaftlichen Diskussion über die pathophysiologischen Zusammenhänge der Dekompressionserkrankung [15-17] eine Empfehlung für die Ruhiglagerung des Opfers eines schweren Tauchunfalls. Bewegungen des Patienten sowie Vibrationen und Erschütterungen können die Menge intravaskulärer Gasblasen erhöhen ([18], • Abb. 1).

Für den Primärtransport gibt es keine prinzipielle Präferenz für ein bestimm- tes Transportmittel. Weiterhin wird empfohlen, bestmöglich Druckreduzierungen während des Transports (Flughöhe, Fahrten über Bergpässe) sowie Erschütterungen (Boot) zu vermeiden.

\section{Hyperbare Sauerstofftherapie}

Die HBO ist bei Dekompressionsunfällen unverändert als Goldstandard anzusehen und derzeit ohne therapeutische Alternative [6]. Das Behandlungsschema entsprechend „US Navy Treatment Table 6“ 


\section{US Navy Treatment Table 6 \\ Modifiziert nach SchiffMedlnstM/GTÜM}

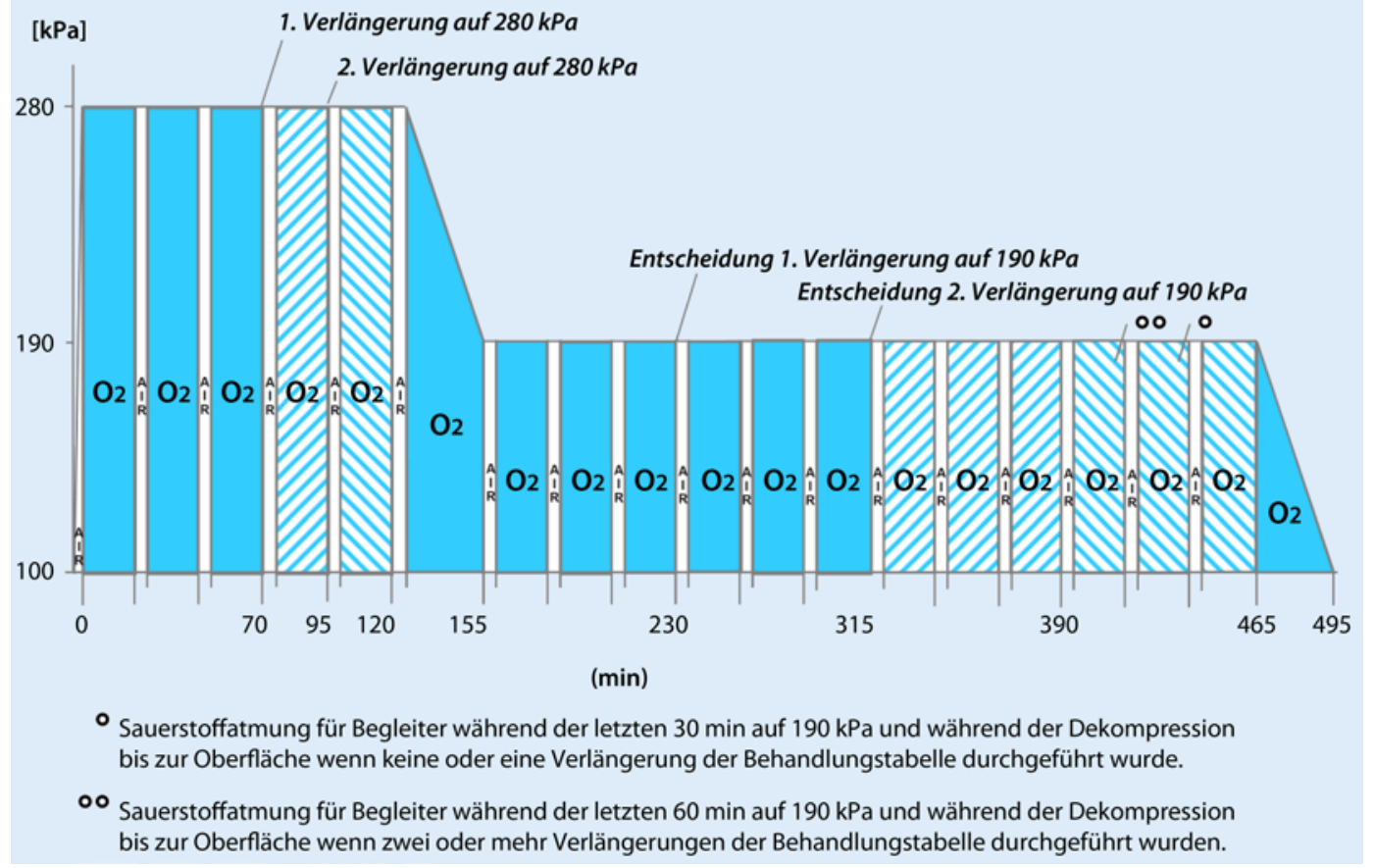

Abb. $3<_{\text {„US Navy Treat- }}$ ment Table 6". $D C I_{\text {II }}$ decompression illness" (Dekompressionserkrankung). (Modifiziert nach SchiffMedlnstM/GTÜM) wird unabhängig von dem Atemgas, das der verunfallten Tauchers verwendet hat, empfohlen ([3], - Abb. 2 und 3). Weiterhin soll eine frühestmögliche $\mathrm{HBO}$ erreicht werden. Die berichteten Behandlungsergebnisse beschreiben die vollständige Rückbildung der Symptome in 67$75,6 \%$ der Fälle in Abhängigkeit von dem Beginn der Rekompression nach Auftreten der ersten Symptome [19, 20]. Erfolgreiche HBO-Behandlungsergebnisse viele Stunden nach Symptombeginn verdeutlichen nach Einschätzung der Autoren der Leitlinie v. a. die komplexe Erkrankung mit systemischen inflammatorischen Reaktionen gegenüber der pathophysiologischen Annahme einer alleinigen „Gasblasenerkrankung“.

Während des Primärtransports soll die Atmung von $100 \%$ igem Sauerstoff ohne Pause bis zum Erreichen der Behandlungskammer weitergeführt werden.

Die HBO soll von einem tauchmedizinisch fortgebildeten Arzt $^{1}$ indiziert und

\footnotetext{
${ }^{1}$ Die Qualifikation soll mindestens den Weiterbildungsinhalten des "diving medicine physician" entsprechen (http://www.gtuem.org, http:// www.suhms.org oder http://www.edtc.org).
}

durchgeführt werden. Die Druckkammerbehandlung kann bei vollständigem Rückgang der nachfolgend aufgeführten Symptome innerhalb der ersten $10 \mathrm{~min}$ der hyperbaren Oxygenation bei $280 \mathrm{kPa}$ verkürzt werden:

- konstitutionelle bzw. unspezifische Symptome - ausgeprägte Müdigkeit,

- kutane Symptome - Hautveränderungen,

- lymphatische Symptome - lokale Schwellung,

- muskuloskelettale Symptome - Gelenk- und Gliederschmerzen,

- leichte peripher-neurologische subjektive sensorische Störungen ohne objektivierbare pathologische Befunde.

In diesen Fällen kann die Behandlung verkürzt entsprechend einer „US Navy Treatment Table 5 “ oder analogen Tabellen beendet werden. Es dürfen jedoch keine zusätzlichen schweren Symptome vorliegen oder vorgelegen haben.

Sind nach der ersten Druckkammerbehandlung noch Symptome vorhanden, soll sich innerhalb von 24 h eine Folgebehandlung anschließen. Wenn zwischen den Behandlungen vor Ort insbesondere an entlegenen Destinationen keine stationäre medizinische Versorgung möglich ist, muss ein Transport in ein entsprechend ausgestattetes Behandlungszentrum erfolgen. Die Wahl des Transportmittels erfolgt unter Abwägung des Patientenzustands, von Transportstrecke und Transportzeit sowie des möglichen „Transportmittels“. Aus Sicht der Leitliniengruppe kann hier kein prinzipielles Vorgehen empfohlen werden. Die Planung kann und sollte in Absprache mit einer der taucherärztlichen Telefonberatungen erfolgen.

Für eine eindeutige Empfehlung der Zeit nach der ersten HBO bis zu einer Verlegung mit dem Flugzeug fehlen systematisch dokumentierte Behandlungsergebnisse [21].

\section{Versorgungssituation in Deutschland}

Der gemeinsame Bundesausschuss hat die HBO für die Dekompressionserkrankung und die arterielle Gasembolie gemäß den Kriterien des $\$ 137$ c des Fünften Buches Sozialgesetzbuch (SGB V) als aus- 
reichend, zweckmäßig und wirtschaftlich beurteilt. Statistiken über die Häufigkeit von Dekompressionsunfällen in Deutschland stehen nur eingeschränkt zur Verfügung. In dem Abschlussbericht des Ausschusses Krankenhaus wurde die Zahl schwerster Tauchunfälle in Deutschland auf über 200/Jahr geschätzt. Die Möglichkeit einer HBO für eine 24-h-Versorgung an 365 Tagen besteht in Deutschland derzeit an 7 Behandlungseinrichtungen [22]. Nur diese Notfallzentren geben eine kontinuierliche Erreichbarkeit und die Möglichkeit für eine intensivmedizinische Behandlung während und nach der HBO an. Andere Druckkammeranlagen in Deutschland können entweder keine fortwährende Rufbereitschaft außerhalb der täglichen Routinedienstzeit oder keine intensivmedizinische Therapie gewährleisten.

Eine wesentliche Schwierigkeit im Hinblick auf die Vorhaltung und die Finanzierung besteht darin, dass viele Druckkammern nicht in eine Klinik der höheren Versorgungsstufe integriert und Mehrkosten durch Sonderentgelte außerhalb des Klassifikationssystems für ein pauschaliertes Abrechnungsverfahren nicht vereinbart sind.

Neben der notwendigen Sicherstellung und Beauftragung der $\mathrm{HBO}$ in den Bundesländern ist die Einrichtung einer zentralen Vermittlung der HBO-Behandlungsplätze, vergleichbar der „Zentralen Anlaufstelle für die Vermittlung von Betten für Schwerbrandverletzte" (ZA Schwerbrandverletzte) denkbar. Diese Entwicklung wird von den zuständigen Fachgesellschaften, der GTÜM, der DIVI Sektion Hyperbarmedizin und dem VDD verfolgt und soll mit der Rettungsleitstelle einer Berufsfeuerwehr umgesetzt werden. Die Probleme der Tauchunfallversorgung in Deutschland, insbesondere in Verbindung mit der Verfügbarkeit einer HBO, werden seit 3 Jahren intensiv diskutiert [23]. Die erste Zielsetzung einer verbesserten Organisation mit einer besseren Kommunikation der Rettungsleitstellen scheint in absehbarer Zeit erreichbar zu werden.

Die in der Leitlinie angegebenen taucherärztlichen Telefonberatungen gewährleisten zudem eine gute Qualität und 24-h-Verfügbarkeit.
Für den präklinisch tätigen Notarzt bleibt die Empfehlung des Transports in die nächste geeignete Notfallaufnahme eines Krankenhauses. Der Rettungsdienst und der Arzt in der Notfallaufnahme eines Krankenhauses sollen über die zuständige Rettungsleitstelle sowohl die Vermittlung einer "taucherärztlichen Telefonberatung" als auch die Zuweisung einer übernehmenden einsatzbereiten Druckkammer anstreben. Aufgrund der unzureichenden Versorgungssituation werden weiterhin zeitaufwendige Sekundärverlegungen notwendig sein.

Zukünftig sollte von den Fachgesellschaften die Etablierung eines bundesweiten Registers von HBO-Notfallbehandlungen angestrebt werden. Nur auf diesem Weg lassen sich eine Qualitätssicherung der hyperbarmedizinischen Versorgung und wissenschaftliche Beiträge zur Versorgungsforschung erreichen.

\section{Korrespondenzadresse}

PD Dr. B. Jüttner M.A.

Klinik für Anästhesiologie und Intensivmedizin Medizinische Hochschule Hannover Carl-Neuberg-Str. 1, 30625 Hannover juettner.bjoern@mh-hannover.de

\section{Einhaltung ethischer Richtlinien}

Interessenkonflikt. Die Darlegung von möglichen Interessenkonflikten erfolgte für die Mitglieder der Leitliniengruppe B. Jüttner, C. Wölfel, H. Liedtke, K. Meyne, H. Werr, T. Bräuer, M. Kemmerer, G. Schmeißer, T. Piepho, O. Müller und H. Schöppenthau mit dem von der AWMF vorgeschlagenen Formblatt. Die Angaben sind in dem „Leitlinienreport zu der methodischen Vorgehensweise bei der Entwicklung der Leitlinie Tauchunfall der GTÜM e.V. (AWMF-Register-Nr.: 072-001) in Zusammenarbeit mit der SUHMS in der Schweiz" aufgeführt.

Dieser Beitrag beinhaltet keine Studien an Menschen oder Tieren.

\section{Literatur}

1. Gesellschaft für Tauch- und Überdruckmedizin (GTÜM e. V.). Leitlinie Tauchunfall 2014-2017. http://www.awmf.org/leitlinien/detail/l//072-001. html. Zugegriffen: 02. Apr. 2015

2. Mitchell SJ, Doolette DJ, Wacholz CJ, Vann RD (2006) Management of mild or marginal decompression illness in remote locations workshop proceedings. DHM 3:152-155

3. Vann RD, Butler FK, Mitchell SJ, Moon RE (2011) Decompression illness. Lancet 377:153-164

4. Petri NM, Andrić D (2003) Differential diagnostic problems of decompression sickness-examples from specialist physicians' practices in diving medicine. Arch Med Res 34:26-30
5. Sundal E, Grønning M, Troland K, Irgens A, Aanderud L, Thorsen E (2011) Risk of misclassification of decompression sickness. Int Marit Health 62:17-19

6. Bennett MH, Lehm JP, Mitchell SJ, Wasiak J (2012) Recompression and adjunctive therapy for decompression illness. Cochrane Database Syst Rev 5:CD005277

7. Böttcher F, Jüttner B, Krause A, Rocha M, Koppert W (2012) Case report: fatal diving-accident. Or: accident while diving? Anaesthesist 61:137-142

8. Gempp E, Blatteau JE, Stephant E, Pontier JM, Constantin P, Peny C (2008) MRI findings and clinical outcome in 45 divers with spinal cord decompression sickness. Aviat Space Environ Med 79:1112-1116

9. Hyldegaard O, Moller M, Madsen J (1991) Effect of $\mathrm{He}-\mathrm{O} 2, \mathrm{O} 2$, and $\mathrm{N} 2 \mathrm{O}-\mathrm{O} 2$ breathing on injected bubbles in spinal white matter. Undersea Biomed Res 18:361-371

10. Longphre JM, Denoble PJ, Moon RE, Vann RD, Freiberger JJ (2007) First aid normobaric oxygen for the treatment of recreational diving injuries. Undersea Hyperb Med 34:43-49

11. Edmonds C, Lippmann J, Lockley S, Wolfers D (2012) Scuba divers' pulmonary oedema: recurrences and fatalities. Diving Hyperb Med 42:40-44

12. Taylor BJ, Carlson AR, Miller AD, Johnson BD (2014) Exercise-induced interstitial pulmonary edema at sea-level in young and old healthy humans. Respir Physiol Neurobiol 191:17-25

13. Van Allen CM, Hrdina LS, Clark J (1929) Air embolism from the pulmonary vein. Arch Surg 19:567599

14. Gorman DF, Browning DM, Parsons DW et al (1987) Distribution of arterial gas emboli in the pial circulation. SPUMS J 17:101-115

15. Thom SR, Milovanova TN, Bogush M, Yang M, Bhopale VM, Pollock NW, Ljubkovic M, Denoble P, Madden D, Lozo M, Dujic Z (2013) Bubbles, microparticles, and neutrophil activation: changes with exercise level and breathing gas during open-water SCUBA diving. J Appl Physiol 114:1396-1405

16. Papadopoulou V, Tang MX, Balestra C, Eckersley RJ, Karapantsios TD (2014) Circulatory bubble dynamics: from physical to biological aspects. Adv Colloid Interface Sci 206:239-249

17. Balestra C (2014) The lymphatic pathway for microbubbles. Diving Hyperb Med 44:1

18. Papadopoulou V, Eckersley RJ, Balestra C, Karapantsios TD, Tang MX (2013) A critical review of physiological bubble formation in hyperbaric decompression. Adv Colloid Interface Sci 191192:22-30

19. Gempp E, Blatteau JE (2010) Risk factors and treatment outcome in scuba divers with spinal cord decompression sickness. J Crit Care 25:236-242

20. Xu W, Liu W, Huang G, Zou Z, Cai Z, Xu W (2012) Decompression illness: clinical aspects of 5278 consecutive cases treated in a single hyperbaric unit. PLoS One 7:e50079

21. Dowse ML, Barnes R, Smerdon G, Bryson P (2005) Time to fly after hyperbaric chamber treatment for decompression illness: current recommendations. SPUMS J 35:67-70

22. Notfallzentren für Tauchunfälle und andere (Notfall-) Indikationen für die hyperbare Sauerstofftherapie. http://www.gtuem.org. Zugegriffen: 02. Apr. 2015

23. Jüttner B, Bartmann H (2014) Hyperbare Sauerstofftherapie. Arbeitsmed Sozialmed Umweltmed 49:895-899 